\title{
DLTS STUDY OF Be-DOPED $p$-TYPE AlGaAs/GaAs MBE LAYERS
}

\author{
J. Szatkowski, E. Placzek-Popko, K. Sierański \\ Institute of Physics, Technical University of Wrocław \\ Wybrzeże Wyspiańskiego 27, 50-370 Wrocław, Poland
}

AND O.P. HANSEN

Ersted Laboratory, University of Copenhagen

Universitetparken 5, 2100 Copenhagen, Denmark

\begin{abstract}
Deep-level transient spectroscopy method was applied to study deep hole traps in $p$-type $\mathrm{Al}_{0.5} \mathrm{Ga}_{0.5} \mathrm{As}$ grown on $\mathrm{GaAs}$ semi-insulating substrate by MBE. Five hole traps labelled by us as $H 0$ to $H_{4}$ were found. For the traps $H 1, H 3$ and $H 4$ thermal activation energies obtained from Arrhenius plots were equal to: $E_{H 1}=0.15 \mathrm{eV}, E_{H 3}=0.4 \mathrm{eV}$, and $E_{H 4}=0.46 \mathrm{eV}$. Hole emission from the trap $\mathrm{H}_{2}$ was electric field dependent with the thermal activation energy extrapolated to zero-field equal to $0.37 \mathrm{eV}$. Capture cross-sections for the traps $H 1$ and $H 4$ were thermally activated with energetic barriers $0.04 \mathrm{eV}$ (for $H 1$ ) and $0.18 \mathrm{eV}$ (for $H 4$ ).
\end{abstract}

PACS numbers: 71.55.-i, 71.55.Eq

\section{Experiment and samples}

In this work we are interested in electrical properties of deep hole traps presented in Be-doped $\mathrm{Al}_{0.5} \mathrm{Ga}_{0.5} \mathrm{As}$ grown by molecular beam epitaxy (MBE). The choice of the Al fraction as 0.5 had its background in previous sample configurations used for the study of two-dimensional hole gases in $\mathrm{Al}_{0.5} \mathrm{Ga}_{0.5} \mathrm{As} / \mathrm{GaAs}$ heterostructures [1].

The samples of $1 \mu \mathrm{m}$ thickness were obtained by MBE growth on a [001] GaAs semi-insulating substrate. The Schottky contacts were made by thermal evaporation of $\mathrm{Al} / \mathrm{Ti} / \mathrm{Au}$ layers of $1 \mathrm{~mm}^{2}$ area whereas the ohmic contacts were realised by thermal evaporation of $\mathrm{Au} / \mathrm{Zn} / \mathrm{Au}$ followed by alloying. Capacitance-voltage $(C-V)$ measurements performed at room temperature yielded linear $C^{-2}-V$ plot. The net acceptor concentration $N_{\mathrm{A}}-N_{\mathrm{D}}$ equal to $(2.0 \pm 0.1) \times 10^{17} \mathrm{~cm}^{-3}$ and built-in voltage $U_{\mathrm{B}}=(1.1 \pm 0.1) \mathrm{V}$ were calculated from the $C-V$ data. Deep-level transient spectroscopy (DLTS) measurements were performed within 77-300 K temperature range. To study electric field influence on emission rate from the traps and temperature dependence of their capture cross-section differential DLTS (DDLTS) mode of operation of the DLS-82E system was applied [2]. 


\section{Results and discussion}

The standard DLTS measurements were carried out for different reverse voltage $U_{\mathrm{R}}$ ranging from -3.5 to $-5 \mathrm{~V}$. Different duration time of filling pulses were used. Usually $20 \mu$ s pulse width was applied. In our DLTS system the DLTS signal minimum takes place at temperature where lock-in frequency, $f$, is related to emission rate by $e_{\mathrm{p}}=2.17 f$.

Five dominant levels, labeled by us as $H 0$ to $H 4$ were detected in all of our samples. The temperature position of the DLTS signal minimum corresponding to $H 1, H 3$, and $H 4$ levels was independent of the height and duration of a filling pulse. A position of the $H 2$ minimum was dependent on the height of filling pulse indicating electric field enhanced emission rate. Strong temperature dependent capture cross-section was observed for the traps $H 1$ and $H 4$. The $H 0$ trap was not studied in detail as the temperature position of its DLTS peak was below $77 \mathrm{~K}$. The dynamic process of capture and emission of holes by deep traps can be described in terms of a capture cross-section, $\sigma_{\mathrm{p}}$, and emission rate, $e_{\mathrm{p}}$. The activation energy and capture cross-section can be determined from Arrhenius plot, i.e. from the plot of experimentally obtained emission rates divided by square temperature $\left(e_{\mathrm{p}} / T^{2}\right)$ versus reciprocal temperature $(1 / T)$. Arrhenius plots related to the traps $H 1-H 4$ are shown in Fig. 1. For $H_{2}$ trap the Arrhenius plots for higher field $\left(\mathrm{H}_{2}-\mathrm{H}\right)-$

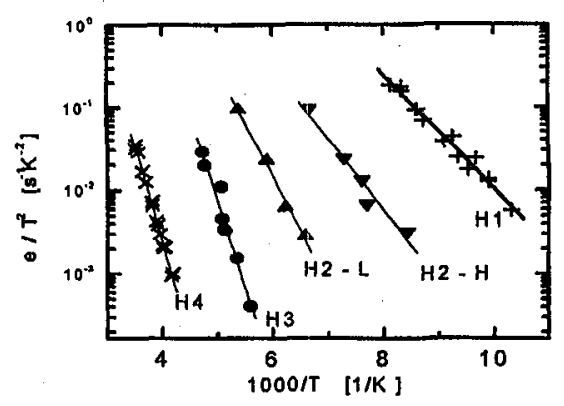

Fig. 1. Arrhenius plots for the traps $H 1, H 2, H 3$, and $H 4$. The straight lines are the best least squares fit to the experimental data.

$4.5 \times 10^{5} \mathrm{~V} / \mathrm{cm}$ and lower field $(H 2-L) 1.8 \times 10^{5} \mathrm{~V} / \mathrm{cm}$ are presented. Activation energy $E_{\mathrm{T}}$ and capture cross-section $\sigma_{\mathrm{p}}$ calculated by the least squares method are summarised in Table I. For $H 2$ trap, from the Arrhenius plots, we obtained $0.22 \mathrm{eV}$ for higher field and $0.26 \mathrm{eV}$ for lower field.

In all samples the traps concentrations are of the order of $10^{14} \mathrm{~cm}^{-3}$. Concentration of $H 1$ is decreasing slightly towards the junction depth (from $5 \times 10^{14} \mathrm{~cm}^{-3}$ to $\left.2 \times 10^{14} \mathrm{~cm}^{-3}\right)$ whereas for the trap $H 4$ it remains nearly constant (about $2 \times 10^{14} \mathrm{~cm}^{-3}$ ) within whole junction region. The profile for $H 3$ trap was not measured as it was very difficult to extract the value of DLTS signal related to these traps from the total DLTS signal. Qualitative comparison of corresponding DLTS signal allows us to evaluate that its concentration is of the order of $10^{13} \mathrm{~cm}^{-3}$. 


\section{TABLE I}

Activation energy $E_{\mathrm{T}}$ and capture cross-section $\sigma_{\mathrm{p}}$ for $H 1, H 2, H 3$, $H 4$ traps.

\begin{tabular}{c|c|c}
\hline \hline Trap & $E_{\mathrm{T}}[\mathrm{eV}]$ & $\sigma_{\mathrm{p}}\left[\mathrm{cm}^{2}\right]$ \\
\hline$H 1$ & 0.15 & $5 \times 10^{-17}$ \\
$H 2-L$ & 0.26 & - \\
$H 2-H$ & 0.22 & - \\
$H 3$ & 0.40 & $3 \times 10^{-14}$ \\
$H 4$ & 0.46 & $6 \times 10^{-16}$
\end{tabular}

We have obtained electric field dependence of emission rates from the trap H2 using DDLTS measurements. We obtained a square root electric field dependence of emission rate, which we interpret as the consequence of the Frenkel-Poole effect. The trap activation energy $E_{\mathrm{T}}$ should depend then on the electric-field intensity $F$ according to the equation

$$
E_{\mathrm{T}}(F)=E_{\mathrm{T} 0}-\alpha F^{1 / 2}
$$

where $E_{\mathrm{T} 0}$ is the activation energy in the bulk (i.e. far away from the space-charge region). The experimentally obtained value of $\alpha$ is equal to $(2.3 \pm 0.1) \times 10^{-4}$ $\left((\mathrm{eV})^{2} \mathrm{~cm} / \mathrm{V}\right)^{1 / 2}$ at all temperatures which agrees excellently with the theoretical one $\left.\alpha=2.2 \times 10^{-4}\left((\mathrm{eV})^{2}\right) \mathrm{cm} / \mathrm{V}\right)^{1 / 2}$. From experimentally obtained value of $\alpha$ we calculated $E_{\mathrm{T} 0}=0.37 \mathrm{eV}$. The electric field dependence of emission rate indicates that carrier emission from the trap has to be accompanied by acquiring a net charge by the defect, i.e. the $H 2$ trap has to be charged upon a hole emission and neutral upon a hole capture. This implies that the trap $H 2$ would then be defined as an acceptor-like level $(0 /-)$ in a $p$-type material.

We measured also the capture cross-section with the method, originally proposed by Henry and Lang [3]. From these measurements for two of the traps $H 1$ and $H 4$ we obtained strong temperature dependence of capture cross-sections. The results are shown in Fig. 2. For both traps capture cross-section $\sigma_{\mathrm{p}}$ is exponentially decreasing function of $1 / T$ :

$$
\sigma_{\mathrm{p}}=\sigma_{\mathrm{p} \infty} \exp \left(-E_{\mathrm{B}} / k T\right)
$$

where $\sigma_{\mathrm{p} \infty}$ is the high-temperature cross-section and $E_{\mathrm{B}}$ is the thermal energy barrier for a hole to be captured. The solid lines in Fig. 2 present the best theoretical fit of Eq. (2) to the experimental data. The values of $E_{\mathrm{B}}$ and $\sigma_{\mathrm{p} \infty}$ yielded from the fit are given in Table II. Energetic barrier for a capture process means strong hole-lattice interaction for both traps $H 1$ and $H 4$. Thermal activation energies obtained from Arrhenius plots are equal to $0.15 \mathrm{eV}$ for $H 1$ and $0.46 \mathrm{eV}$ for $H 4$ (cf. Table I). Subtraction of the barrier energies from these values results in the distance of the $H 1$ and $H 4$ levels from the top of valence band $E_{\mathrm{T}}-E_{\mathrm{B}}$ equal to $0.11 \mathrm{eV}$ and $0.28 \mathrm{eV}$, respectively.

In Fig. 3 we have compared our results $(x=0.5)$ with the results obtained by other authors for different $\mathrm{Al}$ content. In the figure the trap energies are referred 


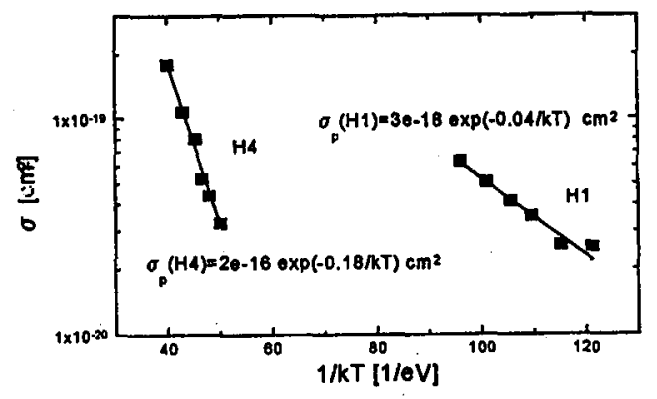

Fig. 2. Temperature dependence of capture cross-sections for the traps $H 1$ and $H 4$.

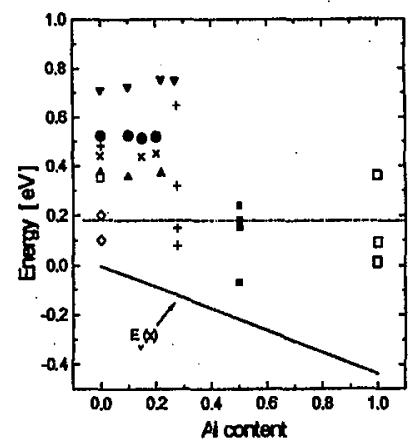

Fig. 3. Trap energies in $\mathrm{Al}_{x} \mathrm{Ga}_{1-x} \mathrm{As}$ as referred with respect to the valence band edge versus $\mathrm{Al}$ content, after taking into account valence band shift with $\mathrm{Al}$ content $x$. Dashed line is only eye-guide. $\bullet$ Fe Lang [4], full $\nabla$ B Lang. [4], $\times$ Cu Lang [4], full $\triangle \mathrm{A}$ Lang [4], $\longrightarrow E_{\mathrm{v}}, \square[5]$, our data, $\diamond[6],+[7], \square[7]$.

\section{TABLE II}

Capture energy $E_{\mathrm{B}}$ infinity temperature capture cross-section $\sigma_{\mathrm{p} \infty}$ and the real trap energy $E_{\mathrm{T}}-E_{\mathrm{B}}$ for $H 1$ and $H 4$ traps.

\begin{tabular}{c|c|c|c}
\hline Trap & $E_{\mathrm{B}}[\mathrm{eV}]$ & $\sigma_{\mathrm{p} \infty}\left[\mathrm{cm}^{2}\right]$ & $E_{\mathrm{T}}-E_{\mathrm{B}}[\mathrm{eV}]$ \\
\hline$H 1$ & 0.04 & $3 \times 10^{-18}$ & 0.11 \\
$H 4$ & 0.18 & $2 \times 10^{-16}$ & 0.28
\end{tabular}

with respect to the valence band edge in $\mathrm{Al}_{x} \mathrm{Ga}_{1-x} \mathrm{As}$ (solid line in Fig. 3) with the energy of the GaAs valence band top assumed as the zero-energy level. For the trap $H 2$ the activation energy in the bulk has been drawn. As it is seen the $H 3$ trap can have the same origin as the trap with $E_{\mathrm{T}}$ close to $0.2 \mathrm{eV}$ observed in [6]. The dotted line presents possible change of the energy of this level with respect to the valence band edge with $\mathrm{Al}$ content. 


\section{Acknowledgments}

MBE-growth and sample processing was made by C.B. Soerensen at the III-V Nano-Lab in Copenhagen.

\section{References}

[1] J. Szatkowski, E. Płaczek-Popko, K. Sierański, O.P. Hansen, in: Proc. Int. Conf. on Advanced Devices and Microsystems, Smolenice (Slovakia) 1996, Ed. T. Lalinsky, Institute of Electrical Engineering, Slovak Academy of Science, Bratislava 1996, p. 69 .

[2] G. Ferenczi, P. Krispin, M. Somogyi, J. Appl. Phys. 54, 3902 (1983).

[3] C.H. Henry, D.V. Lang, Phys. Rev. B 15, 989 (1975).

[4] D.V. Lang, R.A. Logan, L.C. Kimerling, in: Physics of Semiconductors, Proc. 13th Int. Conf. Rome 1976, Ed. F.G. Fumi, North-Holland Publ. Co., Amsterdam 1976, p. 615.

[5] P. Krispin, R. Hey, Mater. Sci. Forum 143-147, 359 (1994).

[6] S. Kalem, G.E. Stillman, Jpn. J. Appl. Phys. 33, 6086 (1994).

[7] A.R. Peaker, F. Saleemi, in: Properties of Aluminium Gallium Arsenide, Ed. S. Adachi, EMIS Datareview Series No. 7, INSPEC, London 1993, p. 269. 